\title{
Unambiguous BPSK-like CSC Method for Galileo Acquisition
}

\author{
Wei-Lung Mao, Chorng-Sii Hwang, \\ Chung-Wen Hung \\ Dept. of Electrical Engineering \\ National Yunlin University of Science and Technology \\ Yunlin County 64002, Taiwan \\ wlmao@yuntech.edu.tw, cshwang@yuntech.edu.tw, \\ wenhung@yuntech.edu.tw
}

\author{
Jyh Sheen, Po-Hung Chen \\ Dept. of Electronic Engineering \\ National Formosa University \\ Yunlin County 632, Taiwan \\ jsheen@nfu.edu.tw,paul@nfu.edu.tw
}

Received: July 16, 2019. Revised: October 3, 2021. Accepted: October 25, 2021. Published: November 23, 2021.

\begin{abstract}
Galileo will be Europe's own Global Navigation Satellite System (GNSS), which is aiming to provide highly accurate and guaranteed positioning services. Galileo E1 system has a code period of $4 \mathrm{~ms}$ which is quadruple that of GPS C/A code. In other words, due to the large number of hypotheses in code phase at acquisition stage, a longer searching time or more hardware resource is required. It is difficult to acquire Galileo signal because of longer code length and the multiple peaks of autocorrelation function of BOC modulation. In this paper, the cyclically shift-and-combine (CSC) and BPSK-like architectures are employed to resolve the unambiguous acquisition for BOC modulation and acquires these satellite signals with hardware complexity reduction. The concept of CSC code is to modify the code structure and shorten the code period such that the acquisition burden can be decreased. Simulation results show that our proposed search algorithm can provide better performances in terms of low hardware complexity for acquiring these satellite signals and detection probability at the low value of CNR.
\end{abstract}

Keywords-Galileo; Global Navigation Satellite System (GNSS); cyclically shift-and-combine (CSC); BPSK-like; CNR.

\section{INTRODUCTION}

Nowadays Global Navigation Satellite System (GNSS) has been widely used in many civil and military applications. In 2004, the EU and US make an agreement to establish a common baseline signal BOC $[1,4,5]$ for the Galileo OS and the modernized civil GPS signal on the L1 frequency. The BOC modulation resulting split spectrum signal effectively enables frequency sharing, while providing attributes that include simple implementation, good spectral efficiency, high accuracy, and enhanced multipath resolution. However, BOC modulation brings some drawbacks for multiple peaks, which complicates signal acquisition process of miss-detection or wrong peak selection being higher. In order to avoid the ambiguities of the absolute value of ACF, the unambiguous BPSK-like algorithm, which can be seen as a superposition of two BPSK modulated signals, is employed to solve this difficulty. The CSC method is also presented to reduce the search space of code phases and acquisition computation complexities in this paper. There have been few studies on the Galileo satellite system design under distinct acquisition method environments. Several techniques are proposed to solve caused by the BOC modulation problems [1, 2]. However, the search space is too complicated and time-consuming problem not yet resolved. The only reduce the search complexity to the current references commonly used method is to change the structure of its spreading code, such as the CSC [3]. Although the orthogonality of the modified code will not be as good as the original code, the modified CSC code can indeed correctly acquire the satellite and capture the code-phase information [3]. Also, the BPSK-like technique is used to eliminate the ambiguity of ACF for BOC signal modulation.

Once the acquisition process starts, the Doppler induced frequency offset of the received signal is compensated by a number controlled oscillator (NCO) with a preset Doppler bin. After the compensation process for the frequency offset is finished, the original spreading code is cyclically shifted by $1 / 4$ its length for four groups and then four groups cyclically shifted are combined or added to form a CSC code where the CSC code is original spreading code by half its length. In this step, the band-pass filtered BOC signals with the BPSK-like architecture are correlated with CSC code stated above. Then, the parallel FFT/IFFT structures are utilized to perform the frequency domain correlation. When the non-coherent integration processing has been accomplished, the maximal peak out of $2 \mathrm{~ms}$ search results of code phases is compared to a detection threshold. If the testing statistic has passed the threshold test, the acquisition is finished and can be tried to enter the tracking process.

\section{GALILEO Signal MODEL}

The received Galileo E1B OS signal from a satellite of the Galileo system can be represented as $[1,2]$

$$
\begin{aligned}
y(t) & =y_{E 1 b}(t)+\eta(t) \\
& =A_{i} d_{i}\left(t-\tau_{i}\right) c_{i}\left(t-\tau_{i}\right) s c_{i}\left(t-\tau_{i}\right) \\
& \times \cos \left[2 \pi\left(f_{E 1 b}+f_{d}\right) t+\varphi_{i}\right]+\eta(t)
\end{aligned}
$$

where $A_{i}$ is the amplitude of the signal, $d_{i}(t)$ is the navigation data, $\tau_{i}$ is the time delay. $c_{i}(t)$ is the Galileo E1b PRN code, $s c_{i}(t)$ is the sub-carrier of the BOC signal. $f_{E 1 b}$ and $f_{d}$ are 
the E1b frequency and the Doppler shift, respectively. $\varphi_{i}$ is the initial phase of the received signal, and $\eta(t)$ is additive white Gaussian noise with zero mean and the variance $\sigma_{I F}^{2}$.

The received signal is band-pass filtered, amplified, and down-converted. Due to the frequency down-conversion, the spectrum of the signal is shifted to the intermediate frequency (IF) represented as:

$$
\begin{aligned}
y_{I F}[n] & =y_{E 1 b}[n]+\eta[n] \\
& =A_{i} d_{i}\left[n-\tau_{i} / T_{s}\right] c_{i}\left[n-\tau_{i} / T_{s}\right] s c_{i}\left[n-\tau_{i} / T_{s}\right] \\
& \times \cos \left[2 \pi\left(f_{I F}+f_{d}\right) n T_{s}+\varphi_{i}\right]+\eta[n]
\end{aligned}
$$

where $T_{s}=1 / f_{s}$ is the sampling period. A convenient choice is to sample the IF signal with a sampling frequency $f_{s}=2 B_{I F}$, where $B_{I F}$ is the front-end bandwidth. In this case, it is easily shown that the noise variance becomes

$$
\sigma_{I F}^{2}=E\left\{\eta^{2}(t)\right\}=E\left\{\eta^{2}\left(n T_{s}\right)\right\} N_{0} f_{s} / 2 N_{0} B_{I F}
$$

where $N_{0} / 2$ is the power spectral density of $\eta(t)$.

\section{GALILEO SignAl ACQUisition METHOD}

In this section, we are going to explain and analyze the CSC code in detail. Since the hardware complexity of the code acquisition process in the navigation receiver is directly proportional to the length of the spreading code in use. The main intention of the proposed CSC code is to alleviate the hardware complexity by reducing the original code length. The code length reduction is achieved by code division which is a key procedure of generating the CSC code. A divided code means that it is a portion of the original spreading code. However, dividing the spreading code will dilute its orthogonality with respect to the other ones. For spread spectrum systems, if the orthogonality of the spreading codes is worse, the resulting signal detection probability decreases as well. Therefore, ensuring that the divided codes still possess acceptable orthogonality becomes important. It is shown that the CSC code is derived from the divided codes. The idea behind the CSC code emerges from the correlation functions between the received signal and the divided codes. Correlating any of the divided codes with the received signal, the peak location in the correlations is highly related given the targeted satellite is present. Besides, peaks produced by different divided spreading codes are located on positions that depend on how the codes are divided. As a result, if the divided codes are cautiously designed, the positions of the peaks can exactly lie on the same code phase.

Generating the CSC code consists of two procedures, which are cyclically shifted and combined. In the first step, the concept of "cyclically shift" is illustrated in Figure 1 where the original spreading code is cyclically shifted by half its length. It is obvious that the shifted code is identical to the original spreading code except for the starting code phase. Second, the original spreading code and the shifted code are combined or added to form a new one. Finally the CSC code is derived by dividing the combined code into two parts with equal length and retaining the first one. Accordingly, the CSC code includes two divided codes with different code-phase shifts. One divided code is the first half of the original code while the other one is the second half. As shown in Figure 1, the received signal with an initial code phase ' 2 ' is correlated with the CSC code. Because the CSC code is a linear combination of two divided codes of the original code, the correlation function can be regarded as a superposition of two divided correlation functions. When these two divided codes are cyclically shifted to left by 2 , both of them will be partially matched to the incoming signal. Therefore, the locations of their correlation peaks will overlap and their gains can be combined. In other words, the CSC code can be used to find the correct code phase of the received signal. It is accomplished by cyclically shifting the original spreading code four times and combining them as demonstrated in Fig. 2, where we see the code phases that required examination are only $1 / 4$ of its original code length. Mathematically, the modified CSC code $z_{i}$ can be expressed as

$$
z_{i}=\left[\begin{array}{llll}
z_{i, 0} & z_{i, 1} & \cdots & z_{i, N_{c}^{\prime}-1}
\end{array}\right]^{T}=x_{i 1}+x_{i 2}+x_{i 3}+x_{i 4}
$$

where $N_{C}^{\prime}=\frac{N_{C}}{4}$ and

$$
\begin{gathered}
x_{i 1}=\left[\begin{array}{llll}
x_{i, 0} & x_{i, 1} & \cdots & x_{i, \frac{N_{c}}{4}-1}
\end{array}\right]^{T} \\
x_{i 2}=\left[\begin{array}{llll}
x_{i, \frac{N_{c}}{4}} & x_{i, \frac{N_{c}}{4}+1} & \cdots & x_{i, \frac{N_{c}}{2}-1}
\end{array}\right] \\
x_{i 3}=\left[\begin{array}{llll}
x_{i, \frac{N_{c}}{2}} & x_{i, \frac{N_{c}}{2}+1} & \cdots & x_{i, \frac{3 N_{c}}{4}-1}
\end{array}\right] \\
x_{i 4}=\left[\begin{array}{llll}
x_{i, \frac{3 N_{c}}{4}} & x_{i, \frac{3 N_{c}}{4}+1} & \cdots & x_{i, N_{c}-1}
\end{array}\right]
\end{gathered}
$$

The corresponding CSC code correlation is given by

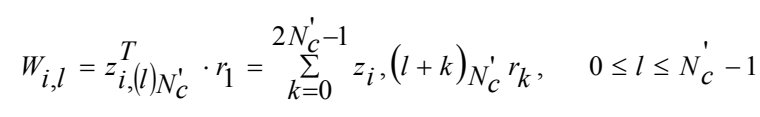

According to (6), there are two periods of the modified CSC code within this interval. The reason for doing so is to maintain the correlation gain. Adopting a similar methodology as for the original CSC code, the correlation results of the modified CSC code can be given by

$$
W_{i, l}=U_{i, l, 1}+I
$$


with $0 \leq l \leq \frac{N_{C}}{4}-l$

where $l$ is the required search range. Once the peak has been identified, a verification of the correct code phase is performed. This time for the modified CSC code, however, checks of four different code phases are indispensable, but these four code phases are not completely independent but obey

$$
l=\left(l+N_{c}^{\prime}\right)_{N_{c}^{\prime}}=\left(l+2 N_{c}^{\prime}\right)_{N_{c}^{\prime}}=\left(l+3 N_{c}^{\prime}\right)_{N_{c}^{\prime}}
$$

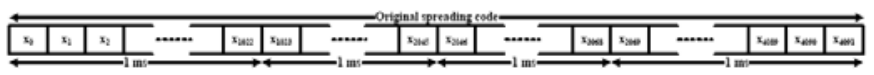

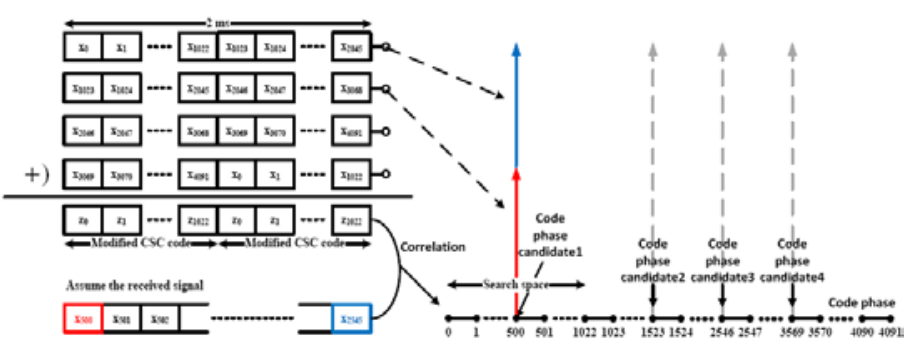

Fig.1. The modified CSC code generation for Galileo E1B signal.

In previous paragraphs the modified CSC code is derived by dissecting the original codes into four different sections. Nevertheless, the formation of the CSC code is not limited to what we have exemplified. A generalized CSC code can be constructed as:

1) Cyclically shift the original spreading code $L$ times such that the initial code phase of them is $0, N_{C} / L, 2 N_{C} / L, \ldots(L-1) N_{C} / L$ chips, respectively. $N_{C}$ is the length of the original spreading codes.

2) Combine or add all of these cyclically shifted codes.

3) Divide the combined code into $L$ successive sections, and the first one is the CSC code with length $N_{C}^{\prime}=N_{C} / L$.

In the steps above, the parameter $L$ must be a common divisor of $N_{C}$ so that only one peak exists within the reduced search space. The generalized CSC code contains $L$-section of the original spreading code simultaneously, so in a sense, the code phase of the received signal is tested in parallel by $L$ different cyclically shifted codes, and hence the acquisition time can be reduced. In fact, by using the CSC code, the search space of the code phase will always be $N_{C} / L$ but the verification on code phase $0, l, l+N_{C}^{\prime}, l+N_{C}^{\prime}, \ldots, l+(L-1) N_{C}^{\prime}$ is necessary. Although the search space of the code phase can be shortened as L increases, the self-interference increases and the correlation peak becomes obscure. Therefore, there is a traded-off between the acquisition time and the correlation performance. In practice, Fig. 4 illustrates how to use the modified CSC code, where the original code length $N_{C}=4096, L=4$, and the modified CSC code length $N_{C}^{\prime}=1023$. The initial code phase of the received signal is at 500. After dispreading operation, the correlation peak is located on 500 , but all the other code phase candidates, i.e. 1523,2546 , and 3569 are also required to be verified.

\section{THE BPSK-LIKE METHOD}

\section{A. Time domain method}

The BOC modulation presents a high degree of spectral separation from conventional signals. But the corresponding acquisition method becomes more complicated. The BPSK-like method [1] consists in considering the received $\mathrm{BOC}(\mathrm{N}, \mathrm{M})$ signal as the sum of two BPSK(M) signals with carrier frequency symmetrically positioned on each side of the BOC carrier frequency. Two correlation channel are generated and the corresponding filtered signals are placed at $f_{I F}+f_{s c}$ and $f_{I F}-f_{s c}$, respectively. To obtain a correlation function whose shape is unambiguous, the BPSK-like method uses only one intermediate frequency with a bandwidth including the two principal lobes of the spectrum. The principle of this method is illustrated in Figure2.

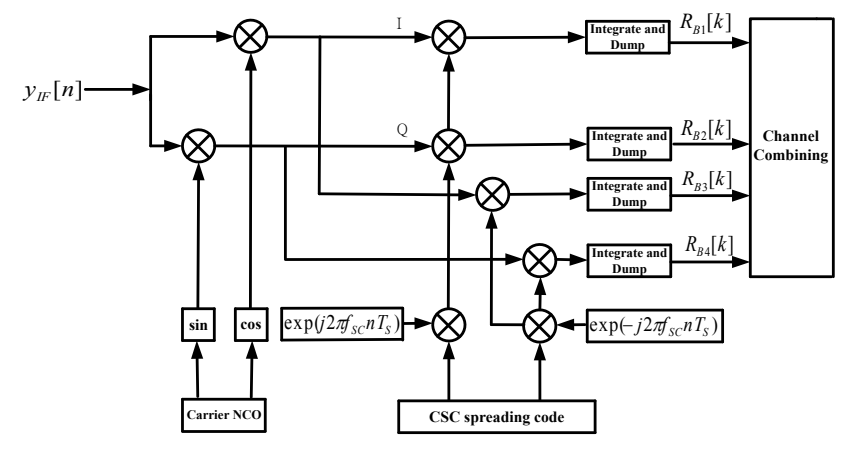

Fig.2. Principle of the time domain BPSK-like method.

At the output of the correlation, the correlation value between the incoming signal and BPSK-like architecture can be represented as

$$
\begin{aligned}
& H_{I}[n]=\cos \left[2 \pi\left(f_{I F}+f_{d}^{\prime}\right) n T_{S}+\varphi_{k}^{\prime}\right] z\left[n-\tau_{i}^{\prime} / T_{S}\right] \exp \left(j 2 \pi f_{S C} n T_{S}\right) \\
& L_{I}[n]=\cos \left[2 \pi\left(f_{I F}+f_{d}^{\prime}\right) n T_{S}+\varphi_{k}^{\prime}\right] z\left[n-\tau_{i}^{\prime} / T_{S}\right] \exp \left(-j 2 \pi f_{S C} n T_{S}\right) \\
& H_{Q}[n]=\sin \left[2 \pi\left(f_{I F}+f_{d}^{\prime}\right) n T_{S}+\varphi_{k}^{\prime}\right] z\left[n-\tau_{i}^{\prime} / T_{S}\right] \exp \left(j 2 \pi f_{S C} n T_{S}\right) \\
& L_{Q}[n]=\sin \left[2 \pi\left(f_{I F}+f_{d}^{\prime}\right) n T_{S}+\varphi_{k}^{\prime}\right] z\left[n-\tau_{i}^{\prime} / T_{S}\right] \exp \left(-j 2 \pi f_{S C} n T_{S}\right)
\end{aligned}
$$

where $\mathrm{z}\left[n-\tau_{i}^{\prime} / T_{S}\right]$ is the CSC spreading code proposed in Eq. (24). The integration and dump function blocks sum up their input signals $\mathrm{y}_{\mathrm{IF}}[\mathrm{n}] H_{I}[\mathrm{n}]$ and $\mathrm{y}_{\mathrm{IF}}[\mathrm{n}] H_{Q}[\mathrm{n}]$ for I-phase and Qphase channels, respectively. Then the outputs of the digital accumulator becomes

$$
\begin{aligned}
& R_{B 1}[k]=\frac{1}{\ell} \sum_{i=0}^{\ell-1}\left\{y_{I F}[n] H_{I}[n]\right\}=y_{I H}[k]+\eta_{I H}[k] \\
& R_{B 2}[k]=\frac{1}{\ell} \sum_{i=0}^{\ell-1}\left\{y_{I F}[n] H_{Q}[n]\right\}=y_{Q H}[k]+\eta_{Q H}[k] \\
& R_{B 3}[k]=\frac{1}{\ell} \sum_{i=0}^{\ell-1}\left\{y_{I F}[n] L_{I}[n]\right\}=y_{I L}[k]+\eta_{I L}[k]
\end{aligned}
$$


$R_{B 4}[k]=\frac{1}{\ell} \sum_{i=0}^{\ell-1}\left\{y_{I F}[n] L_{Q}[n]\right\}=y_{Q L}[k]+\eta_{Q L}[k]$

where $\ell=\mathrm{T}_{\mathrm{C}} / \mathrm{T}_{\mathrm{S}}$ is the number of samples in coherent integration, $\mathrm{T}_{\mathrm{C}}$ is the coherent integration time. The autocorrelation function of received signal components are given as

$y_{I H}[k]=\frac{1}{\ell} \sum_{i=0}^{\ell-1}\left\{\mathrm{y}_{\mathrm{E} 1 \mathrm{~b}}[\mathrm{n}] H_{I}[n]\right\}=\frac{A_{i}}{2} d_{i} R_{H}\left[\Delta \tau / T_{S}\right] \operatorname{sinc}\left(\pi \Delta f_{d} T_{C}\right) \cos \left(\Delta \varphi_{k}\right)$

$y_{Q H}[k]=\frac{1}{\ell} \sum_{i=0}^{\ell-1}\left\{\mathrm{y}_{\mathrm{E} 1 \mathrm{~b}}[\mathrm{n}] H_{Q}[n]\right\}=\frac{A_{i}}{2} d_{i} R_{H}\left[\Delta \tau / T_{S}\right] \operatorname{sinc}\left(\pi \Delta f_{d} T_{C}\right) \sin \left(\Delta \varphi_{k}\right)$

$y_{I L}[k]=\frac{1}{\ell} \sum_{i=0}^{\ell-1}\left\{\mathrm{y}_{\mathrm{E} 1 \mathrm{~b}}[\mathrm{n}] L_{I}[n]\right\}=\frac{A_{i}}{2} d_{i} R_{L}\left[\Delta \tau / T_{S}\right] \operatorname{sinc}\left(\pi \Delta f_{d} T_{C}\right) \cos \left(\Delta \varphi_{k}\right)$

$y_{Q L}[k]=\frac{1}{\ell} \sum_{i=0}^{\ell-1}\left\{\mathrm{y}_{\mathrm{E} 1 \mathrm{~b}}[\mathrm{n}] L_{Q}[n]\right\}=\frac{A_{i}}{2} d_{i} R_{L}\left[\Delta \tau / T_{S}\right] \operatorname{sinc}\left(\pi \Delta f_{d} T_{C}\right) \sin \left(\Delta \varphi_{k}\right)$

where $R_{H}\left[\Delta \tau / T_{S}\right]$ represents the autocorrelation function between the received BOC signal and the local CSC code modulated by $\exp \left(j 2 \pi f_{S C} n T_{S}\right)$, and $R_{L}\left[\Delta \tau / T_{S}\right]$ represents the autocorrelation function between the received BOC signal and the local CSC code modulated by $\exp \left(-j 2 \pi f_{S C} n T_{S}\right)$. The noise components are also divided into real and imaginary components. They are

$$
\begin{aligned}
& \eta_{I H}[k]=\frac{1}{L} \sum_{i=0}^{\ell-1}\left\{\eta[n] H_{I}[n]\right\} \\
& \eta_{Q H}[k]=\frac{1}{L} \sum_{i=0}^{\ell-1}\left\{\eta[n] H_{Q}[n]\right\} \\
& \eta_{I L}[k]=\frac{1}{L} \sum_{i=0}^{\ell-1}\left\{\eta[n] L_{I}[n]\right\} \\
& \eta_{Q L}[k]=\frac{1}{L} \sum_{i=0}^{\ell-1}\left\{\eta[n] L_{Q}[n]\right\}
\end{aligned}
$$

The final decision variable is obtained as

$$
S=\left|R_{B 1}[k]\right|^{2}+\left|R_{B 2}[k]\right|^{2}+\left|R_{B 3}[k]\right|^{2}+\left|R_{B 4}[k]\right|^{2}
$$

\section{B. Frequency domain method}

The parallel frequency domain method is developed such that it can achieve less computational burdens simultaneously search multiple code phase process to speed up the acquisition. The conventional time-domain acquisition can compute the autocorrelation function directly, but it takes a high number of operations. Figure 4 shows the architecture of frequency domain BPSK-like CSC method. The convolution process in the time domain is equal to the multiplications in the frequency domain. If $\mathrm{y}_{\mathrm{IF}}[\mathrm{n}]$ is the Galileo received signal, then the correlation outputs for high-side frequency $R_{H}[\mathrm{~m}]$ and lowside frequency $R_{L}[m]$ can be represented as:

$$
\begin{aligned}
& R_{H}[m]=\sum_{i=1}^{L} x[n] z[n] \exp \left(j 2 \pi f_{s c} n T_{s}\right) \\
& =x[n] \otimes\left[z[-n] \exp \left(-j 2 \pi f_{s c} n T_{s}\right)\right] \\
& =F^{-1}\left(F(X[n]) \times F\left(z[n] \exp \left(j 2 \pi f_{s c} n T_{s}\right)\right)\right)
\end{aligned}
$$

$$
\begin{aligned}
& R_{L}[m]=\sum_{i=1}^{L} x[n] z[n] \exp \left(-j 2 \pi f_{s c} n T_{s}\right) \\
& =x[n] \otimes\left[z[-n] \exp \left(+j 2 \pi f_{s c} n T_{s}\right)\right] \\
& =F^{-1}\left(F(X[n]) \times F\left(z[n] \exp \left(-j 2 \pi f_{s c} n T_{s}\right)\right)\right)
\end{aligned}
$$

with $x[n]=I(n)+j Q(n)$

$$
\begin{aligned}
& I(n)=y_{I F}(n) \cos \left[2 \pi\left(\mathrm{f}_{\mathrm{IF}}+\mathrm{f}_{\mathrm{d}}\right) \mathrm{nT}_{\mathrm{s}}+\varphi_{k}^{\prime}\right] \\
& Q(n)=y_{I F}(n) \sin \left[2 \pi\left(\mathrm{f}_{\mathrm{IF}}+\mathrm{f}_{\mathrm{d}}\right) n \mathrm{~T}_{\mathrm{s}}+\varphi_{k}^{\prime}\right]
\end{aligned}
$$

where $R_{H}[m]$ is the upper band correlation output, $R_{L}[m]$ is the lower band correlation output, $z[n]$ is the CSC code, the operator $\otimes$ means the convolution operation, $\mathrm{F}$ is the Fourier transform, and $\mathrm{F}^{-1}$ means the inverse Fourier transform. In order to construct the decimation-in-frequency FFT algorithm, the FFT equation is expressed as:

$$
X[k]=F(x[n])=\sum_{n=0}^{N-1} x[n] W_{N}^{n k} \quad k=0,1, \ldots N-1
$$

with $W_{N}^{n k}=e^{-j 2 \pi(n k / N)}$

where $X[k]$ is the Fourier transform of the received signal $X[n]$. Acquisition in the frequency domain can reduce a large of number of operations and then increase the speed of computation. The final correlation decision output can be obtained as:

$$
R[m]=R_{H}[m]+R_{L}[m]
$$

where $R[m]$ is the combined correlation output for signal acquisition. If a peak is present in the correlation output $R[\mathrm{~m}]$, the index of this peak marks the PRN code phase of the incoming signal.

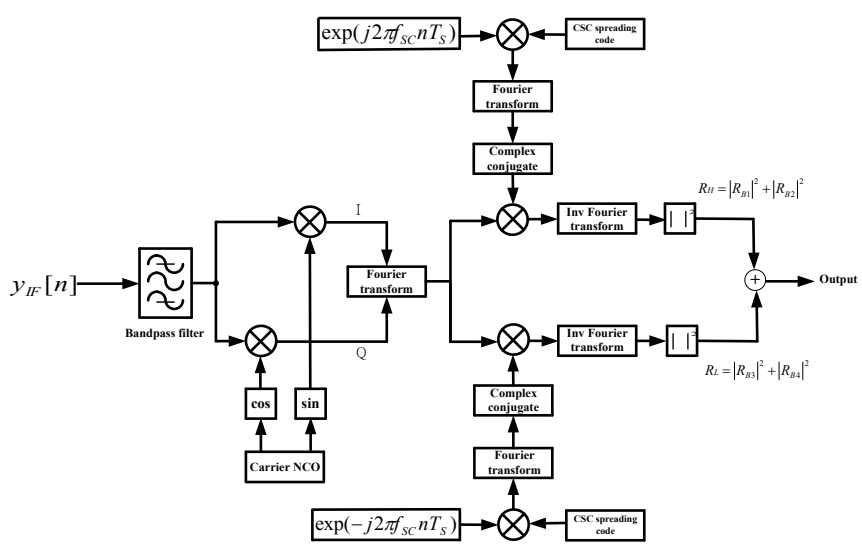

Fig.3. Principle of the frequency domain BPSK-like CSC method. 


\section{PREPARE Your PAPER BEFore STYLING}

In this section, MATLAB tool is use to evaluate the CSC method and BPSK-like structure for original E1b Galileo signal acquisition. The received frequency offset is set to $9.548 \mathrm{MHz}$. Assuming the frequency offset of the received satellite signal is located somewhere between $\pm 5 \mathrm{kHz}$, and the frequency step during the Doppler search is set to $125 \mathrm{~Hz}$.

In Figure 4(a), it shows the two-dimensional autocorrelation function from the received Galileo signal and our proposed CSC method. The integration time is $2 \mathrm{~ms}$ and the SNR of all satellites are set as $-20 \mathrm{~dB}$, which represents an appropriate signal quality. In Figure 4(b), if the integration processing has been accomplished, the maximal peak out of 2 ms search results of code phases is compared to a detection threshold. The CSC method is applied here to determine the true code position between the two peaks in the correlation domain. However, if the maximum of them is larger than the threshold, the code phase and the Doppler frequency are fed into the tracking process, while the acquisition unit restarts to search another satellite signal.

It is shown in Figure 5 that the code phase peak is located at the 1554-th chip for different architectures. The ACFs of the BPSK-like method seems to be much higher than the performance of the $\operatorname{SinBOC}(1,1)$ one. It is shown that the sidelobes of the ACF of $\operatorname{SinBOC}(1,1)$ are clearly visible. These sidelobes will cause more challenges to the BOC acquisition process, and the BPSK-like method is utilized here to remove the additional peaks to improve the search stage.

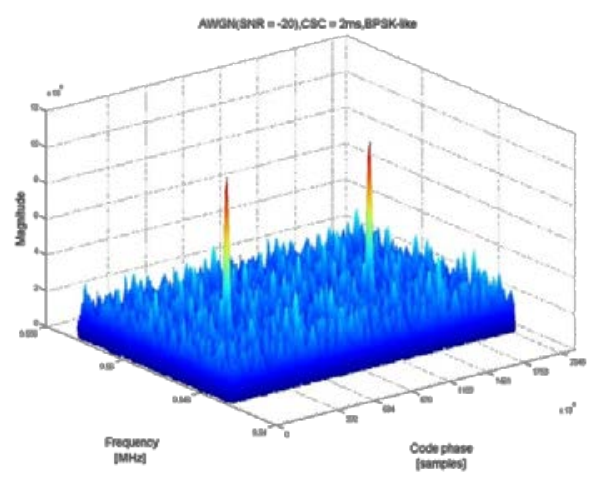

(a)

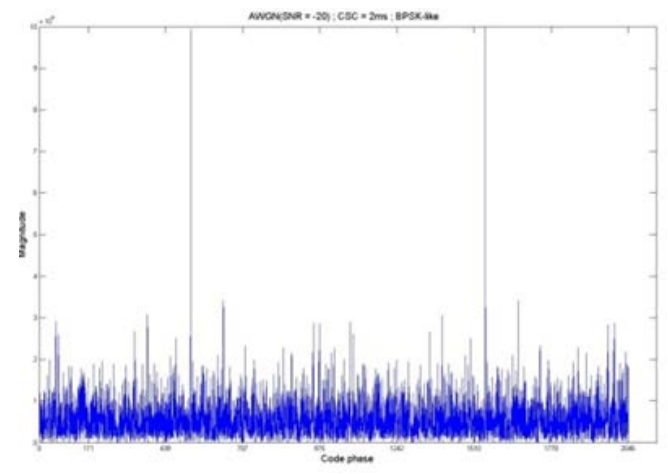

(b)

Fig.4. The acquisition results of BPSK-like architecture (Magnitude Highest: $15 \times 10^{9}$ ) (a) The autocorrelation function of BPSK-like architecture, (b) The autocorrelation function at the zero doppler frequency.
Figure 6 shows the simulation-based histogram for correct and incorrect bins under different CNR environments. A test statistic is calculated in each search window according to the current correlation results. In this simulation, the distribution of the output is based on the chi-square random variable. In Figure 6(a) and Figure 6(b), the noise-only condition is central chi-square distribution, and the Galileo signal with noise is non-central chi-square distribution. In Figure 6(c), it can be observed that CSC method has a better performance under difference CNR. It is shown that the detection probability of CSC method is good enough for Galileo signal acquisition.

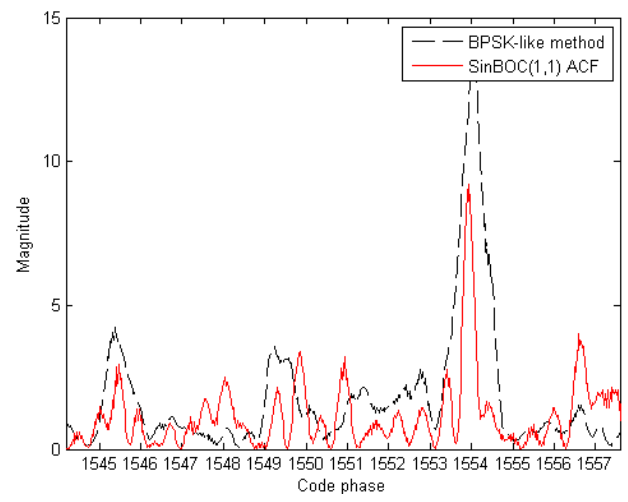

Fig.5. Comparison of the correlation peak in BPSK-like and SinBOC $(1,1)$ architecture.

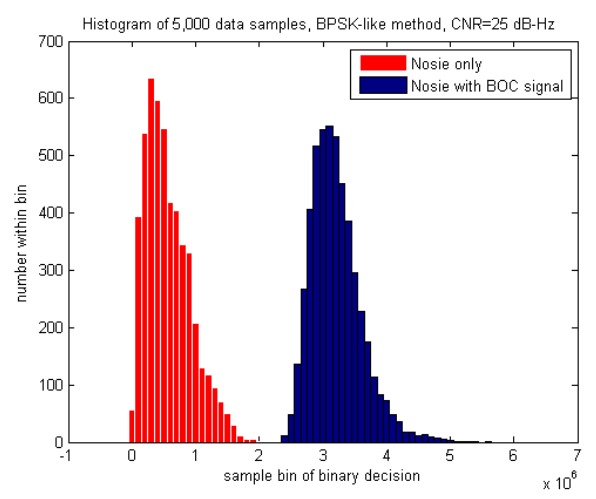

(a) $\mathrm{CNR}=25 \mathrm{~dB}-\mathrm{Hz}$

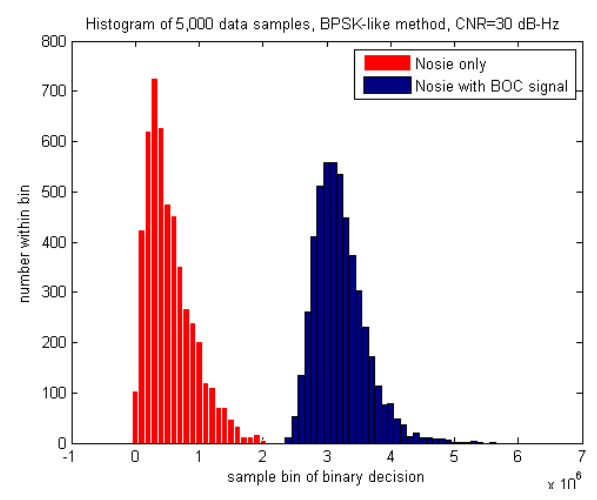

(b) $\mathrm{CNR}=30 \mathrm{~dB}-\mathrm{Hz}$ 


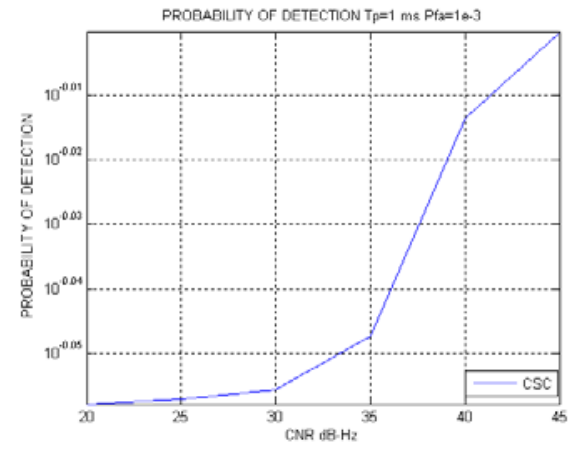

(c) Probability of detection vs. CNR for CSC method

Fig.6. The BPSK-like method acquisition performances, (a) $\mathrm{CNR}=25 \mathrm{~dB}-$ $\mathrm{Hz}$; (b) $\mathrm{CNR}=30 \mathrm{~dB}-\mathrm{Hz}$.(c) Probability of detection vs. CNR with CSC method.

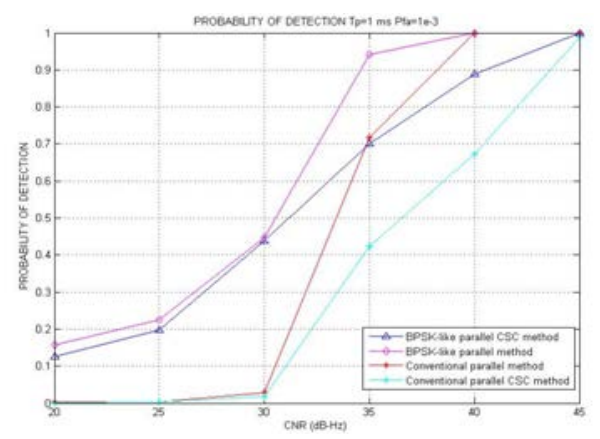

Fig.7. The compasons of detection probability for BPSK-like parallel CSC method.

The simulations done here can assess the performance of our proposed CSC method and parallel BPSK-like method. Four architectures are compared; they are conventional parallel method, BPSK-like parallel method, parallel CSC method, and BPSK-like parallel CSC method. Figure 7 shows the comparisons between these four architectures. It is shown that the BPSK-like method can provide a better performance than the conventional method does under the same CNR conditions. By combining the parallel CSC code, the search space of the code phases shrinks and the corresponding computation burden of the code correlations can be reduced. While CNR is lower than $35 \mathrm{~dB}-\mathrm{Hz}$, the detection rate is better than the conventional parallel method. On the other hand, our BPSK-like parallel CSC code method provides a proper detection probability for high value of CNR, and can be utilized for unambiguous search in BOC modulation system.

\section{CONCLUSIONS}

In this paper, the unambiguous acquisition method using BPSK-like and CSC architecture has been evaluated for Galileo system. The CSC method can be used to reduce the complexity of search procedures, and its performance degradation in correlations has been evaluated. In order to avoid the drawbacks of multiple peaks from BOC modulation, the BPSK-like search structure is used to yield the single correlation peak. Our proposed BPSK-like parallel CSC method can determine the exact phase of the satellite signal and reduce the computation burden. By using the statistical analysis, it shows that BPSK-like parallel CSC method provides the better detection rate than parallel method when CNR is lower than $35 \mathrm{~dB}-\mathrm{Hz}$. It also provides a suitable detection performance for higher CNR conditions. Based on the overall system we proposed, it is aims to accomplish the shorter search time and better detection probability for Galileo satellite signal acquisition.

\section{REFERENCES}

[1] V. Heiries, D. Roviras, L. Ries, V. Calmettes, "Analysis of Non Ambiguous BOC Signal Acquisition performance", ION GNSS 17th International Technical Meeting of the Satellite Division, 21-24 Sept. 2004

[2] Zaixiu Yang, Zhigang Huang, Shengqun Geng, "Unambiguous Acquisition Performance Analysis of BOC $(m, n)$ Signal “, International Conference Information Engineering and Computer Science (ICIECS), pp.1 4, 2009.

[3] Pei-Hsueh Lee, Dong-Hong Liu, Wei-Lung Mao, Hen-WaiTsao, FanRen Chang, "A Novel Low-Complexity Acquisition Method for Next Generation GNSS Signals” ION GNSS 2008, pp. 1507 - 1513, 2008.

[4] Md. Farzan Samad, "Effects of MBOC Modulation on GNSS Acquisition Stage" Tampere University of Technology, master thesis, 2009.

[5] Kai Borre, Dennis M. Akos, NicolajBertelsen, Peter Rinder, SorenHoldt Jensen, "A Software-Defined GPS and Galileo Receiver", 2007.

[6] D. Borio, "A statistical theory for GNSS signal acquisition", Ph.D. dissertation, University of Politecnico di Torino, 2008

[7] J. W. Betz, "The Offset Carrier Modulation for GPS modernization", ION Technical Meeting, 1999.

\section{Creative Commons Attribution License 4.0 (Attribution 4.0 International, CC BY 4.0)}

This article is published under the terms of the Creative Commons Attribution License 4.0

https://creativecommons.org/licenses/by/4.0/deed.en US 